\title{
ÉTUDES ENTOMOLOGIQUES DE POST-TRAITEMENT DANS LE PROGRAMME De LUTte CONTRe L'ONCHOCercose en AfriQue de l'OUest
}

\author{
AGOUA H.*, ALLEY E.S.*, HOUGARD J.-M.**, AKPOBOUA K.L.B.*, BOATIN B.* \& SÉKÉTÉLI A.*
}

Summary : PROCEDURE OF DEFINITIVE CESSATION OF LARVICIDING IN the Onchocerciasis Control Programme in West Africa : entoMOLOGICAL POST-CONTROL STUDIES

In 1989, the Onchocerciasis Control Programme in West Africa started the first larviciding cessation in basins in its original area where the entomological and epidemiological results were considered satisfactory. It was in this context that entomological postcontrol studies were initiated with a view to ensuring that there had been no resumption of transmission of the disease despite the cessation of larviciding and, therefore, a return of the blackflies to their original level. The 18 catching points studied have, overall, given satisfactory results : the pre-control infectivity rates, which ranged between 60 and 90 infective larvae per 1000 parous females, have decreased to less than one infective larva per 1000 parous, the threshold below which the risk of recrudescence of onchocercal infection is considered most unlikely. The only exception is one catching point where the relatively high blackfly infectivity rate (8.9 infective larvae per 1000 parous flies) has led to the resumption of larviciding. The values obtained at two other points, though acceptable (1.08 and 1.16), have made it possible to detect the presence of infected human migrants who have been treated with a microfilaricide, ivermectin, in order to avoid any resumption of onchocerciasis transmission. On the whole, the excellent results revealed by these entomological post-control studies have led to the definitive cessation of larviciding in more than $90 \%$ of the river basins in the original Programme area.

KEY WORDS : onchocerciasis. Simulium damnosum s.I. West Africa. entomological post control. cessation of larviciding.

\section{INTRODUCTION}

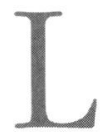

'onchocercose, ou cécité des rivières, est une filariose dermique invalidante qui a constitué durant de longues années non seulement un problème de santé publique, mais aussi un frein au développement socio-économique en Afrique de l'Ouest. Pour lutter contre cette maladie et ses conséquences socio-économiques, un vaste programme de lutte contre l'onchocercose (OCP) ${ }^{1}$, qui couvre actuellement 11 pays $^{2}$, soit environ $1300000 \mathrm{~km}^{2}$, a été mis en place dès 1974. La maladie sévit aux

* Professionnel de l'OMS/OCP,

** Correspondance : J.M. Hougard. Directeur de recherche de l' ORSTOM, mis à disposition de l'OMS/OCP, B.P. 549, Ouagadougou, Burkina Faso.

\section{Résumé}

En 1989, le programme de lutte contre l'onchocercose en Afrique de l'Ouest a procédé dans sa zone initiale aux premiers arrêts de traitements larvicides dans les bassins où les résultats entomologiques et épidémiologiques étaient jugés satisfaisants. C'est dans ce contexte qu'ont été instituées des études entomologiques de post-traitement qui avaient pour but de s'assurer qu'il n'y avait pas de reprise de la transmission de la maladie malgré l'arrêt des traitements et, par conséquent, le retour des simulies à leur niveau initial. Les 18 points étudiés ont donné dans l'ensemble des résultats satisfaisants : les taux d'infectivité, qui variaient avant le début des traitements entre 60 et 90 larves infectantes pour 1000 femelles pares, sont descendus à moins de 1 larve infectante pour 1000 pares, seuil au-dessous duquel le risque de recrudescence de l'infection onchocerquienne est jugé très improbable. Un seul point fait exception où le taux d'infectivité des simulies, relativement élevé 18,9 larves infectantes pour 1000 pares), a entraîné une reprise des traitements larvicides. Les valeurs obtenues en deux autres points, bien qu'acceptables (1,08 et 1, 16), ont permis de déceler la présence de migrants humains infectés qui, pour éviter toute reprise de la transmission de l'onchocercose, ont été traités avec un microfilaricide, l'ivermectine. Dans l'ensemble, les excellents résultats mis en évidence par ces études entomologiques de post-traitement ont conduit à la cessation définitive des épandages larvicides dans plus de $90 \%$ des bassins de l'aire initiale du Programme.

MOTS CLÉS : onchocercose. Simulium damnosum s.I. Afrique de l'Ouest. posttraitement entomologique. arrêt des traitements larvicides.

abords des cours d'eau. Le parasite est une filaire, Onchocerca volvulus (Leuckart, 1893), qui est transmise par un moucheron, diptère nématocère de la famille des Simuliidae. En Afrique de l'Ouest, les espèces vectrices de la maladie appartiennent au complexe Simulium damnosum dont les stades préimaginaux (larves et nymphes) vivent dans les rivières à courant rapide, accrochés aux supports végétaux et rocheux (Philippon, 1977).

Au début des activités de l'OCP, le manque de médicament efficace et utilisable en campagne de masse a contraint les responsables du Programme à mettre en œuvre, dans son aire initiale (fig. 1), une lutte basée

1. OCP (Onchocerciasis Control Programme).

2. Bénin, Burkina-Faso, Côte d'Ivoire, Ghana, Guinée, GuinéeBissau, Mali, Niger, Sénégal, Sierra Leone et Togo. 

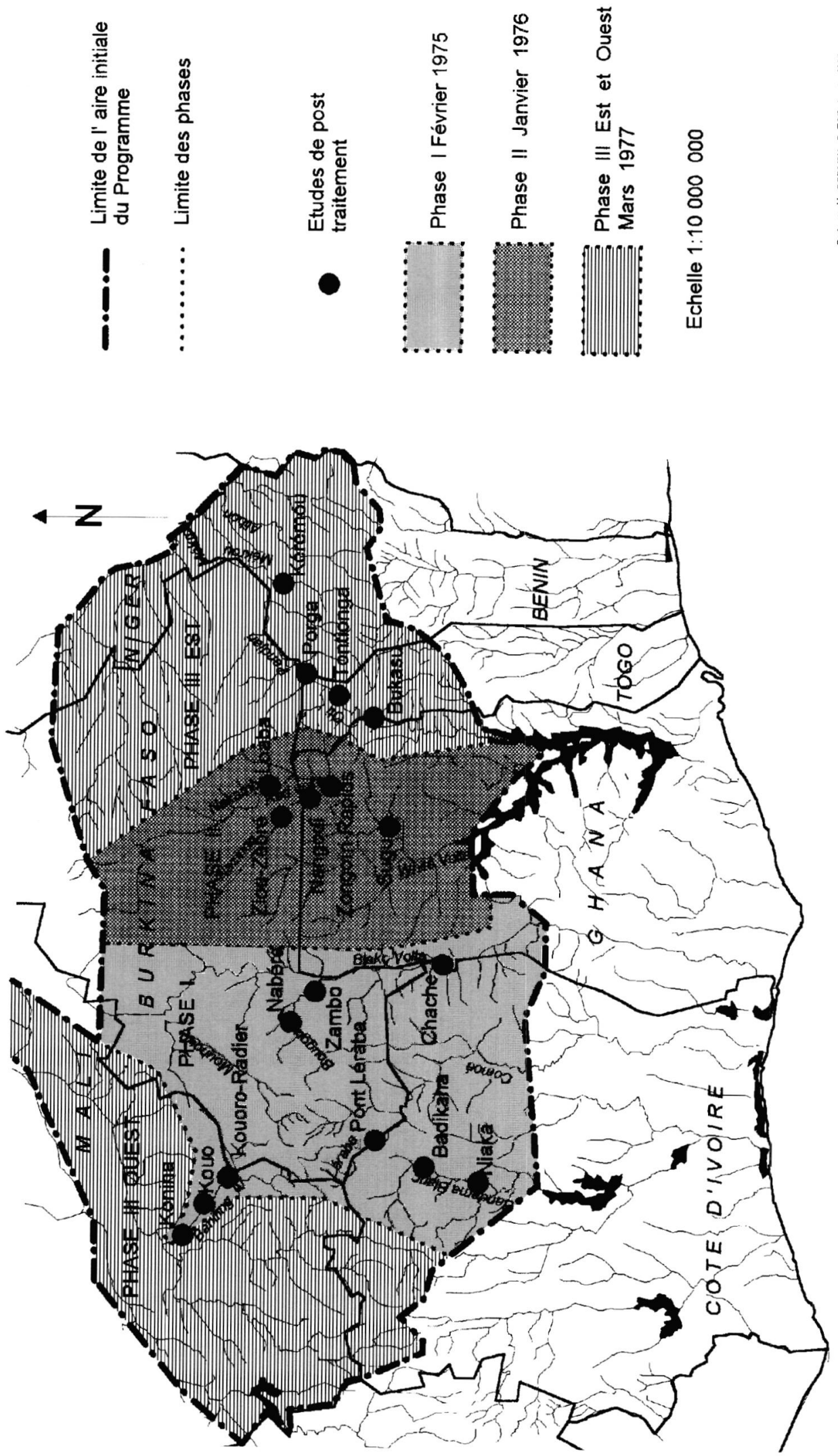

Fig. 1. - Études entomologiques de post-traitement dans la zone initiale du programme de lutte contre l'onchocercose en Afrique de l'Ouest : localisation des points de captures et des rivières concernés. 
exclusivement sur des épandages larvicides, de manière à interrompre la transmission de l'onchocercose pendant une durée supérieure à la longévité du ver adulte (Hougard et al., 1994). Le modèle épidémiologique de la transmission de l'onchocercose "ONCHOSIM" (Plaisier et al., 1990) a montré que la durée de vie moyenne du ver adulte est estimée à 911 ans (Plaisier et al., 1991a). Il a été prédit que $95 \%$ des parasites meurent avant d'atteindre l'âge de 13-14 ans et que 14 ans de lutte antivectorielle efficace à grande échelle suffisent pour abaisser le risque de recrudescence à moins de $1 \%$. En effet, on a noté qu'après ce laps de temps, les résultats obtenus dans la zone initiale du Programme sont excellents du point de vue épidémiologique et permettent l'arrêt des traitements larvicides par suite de l'épuisement du réservoir de parasites. Cependant, les traitements larvicides ne peuvent être arrêtés que si les conditions suivantes sont remplies (Remme et al., 1995): a) la transmission doit avoir été interrompue de façon significative pendant au moins 14 ans de lutte antivectorielle; b) les tendances épidémiologiques observées dans les villages indicateurs de la zone doivent être bonnes et consistantes avec celles prédites par le modèle épidémiologique et c) aucun enfant né après le début des traitements larvicides ne doit être infecté. Cependant, pour vérifier le bien-fondé de la décision d'arrêt des épandages larvicides, l'OCP, sur recommandation de son comité consultatif d'experts (Anonyme, 1990), a entrepris des études entomologiques dites de "post-traitement" visant à déterminer le niveau d'infectivité du vecteur pendant les deux premières années qui suivent l'arrêt des épandages larvicides. Le présent article fait état des résultats de ces études entomologiques qui ont conduit à arrêter définitivement les épandages larvicides sur plus de $90 \%$ des bassins de l'aire initiale du Programme.

\section{MATÉRIEL ET MÉTHODES}

\section{ChOIX DES POINTS DE CAPTURE ET DONNÉES}

\section{ENTOMO-ÉPIDÉMIOLOGIQUES DE BASE}

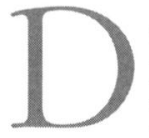

ébutés en février 1975 sur les bassins du Banifing IV, du Bandama-blanc, de la Léraba-Comoé, de la Bougouriba et de la Volta noire, les traitements larvicides dans cette zone se sont étendus par étapes successives en janvier 1976 et mars 1977 sur les bassins du Baoulé, de la Bagoé, de la Volta rouge et blanche, de l'Oti, de l'Alibori et de la Mékrou (fig. 1). Les 18 points de capture retenus pour cette étude ont été sélectionnés sur ces bassins. Il s'agit, en partant de l'ouest de la zone initiale, de Konina, Kouo, et Kouoro-radier sur le Banifing IV, de Niaka et Badikaha sur le Bandama-blanc, de Pont
Léraba sur la Léraba, de Nabéré et Zambo sur la Bougouriba, de Chache sur la Volta Noire, de ZiouZabré et Nangodi sur la Volta rouge, de Sugu, Zongoïri-rapids et Loaba sur la Volta-blanche, de Bukasu et Tontionga sur l'Oti, de Porga sur la Pendjari et de Kérémou sur la Mékrou (fig. 1). Avant le début des traitements iarvicides, plus de la moitié de ces points (54\%) avaient des Potentiels Annuels de Transmission (PAT - voir plus loin) qui variaient entre 800 et 1900 larves infectantes par homme et par an (valeurs associées en zone de savane à des signes cliniques d'hyperendémie onchocerquienne) et des taux d'infectivité pour 1000 femelles pares compris entre 60 et 90 larves infectantes (Quillévéré, 1979). Après 14 à 16 années de lutte antivectorielle ininterrompue, les PAT et les taux d'infectivité ont diminué de façon drastique (Philippon, 1990). À l'exception de Kérémou sur la Mékrou (PAT de 102 et taux d'infectivité de 8,9), les valeurs étaient comprises entre 0 et 41 larves infectantes par homme et par an tandis que les taux d'infectivité ne dépassaient pas une larve infectante pour 1000 femelles pares. Le tableau I, qui récapitule les données épidémiologiques des villages les plus proches des points de post-traitement, confirme les résultats de l'évaluation entomologique. Avant les opérations de lutte, la majorité de ces villages étaient hyperendémiques avec des prévalences de l'infection onchocerquienne allant de $49 \%$ à $84 \%$ et des Charges Microfilariennes des Communautés (CMFC) pouvant atteindre 98 microfilaires par biopsie (la CMFC mesure l'intensité de l'infection et se définit comme étant la moyenne géométrique du nombre de microfilaires trouvées dans les biopsies cutanées des adultes de 20 ans et plus (Remme et al., 1986)). À l'exception de Gbéniki sur la Mékrou, les tendances épidémiologiques ont commencé à décroître rapidement au cours des sept à dix premières années de lutte antivectorielle pour atteindre, après 14 années de lutte, des niveaux tout à fait acceptables (Remme et al., 1990a) compatibles avec un arrêt des traitements larvicides (de 93 à $100 \%$ de réduction de la prévalence) et consistantes avec celles prédites par le modèle épidémiologique ONCHOSIM.

\section{ORGANISATION DES ÉTUDES}

Les études de post-traitement ont débuté en 1990 dans l'aire initiale, une zone de savane où l'onchocercose sévissait avant le début du programme sous sa forme la plus grave, la forme cécitante. Elles ont été effectuées principalement en début et fin de saison des pluies, période où les densités simulidiennes et la transmission sont habituellement les plus élevées. Sur chacun des points retenus, on effectue quatre journées de capture par semaine. En vue de disposer de données comparables à celles d'avant les études de 


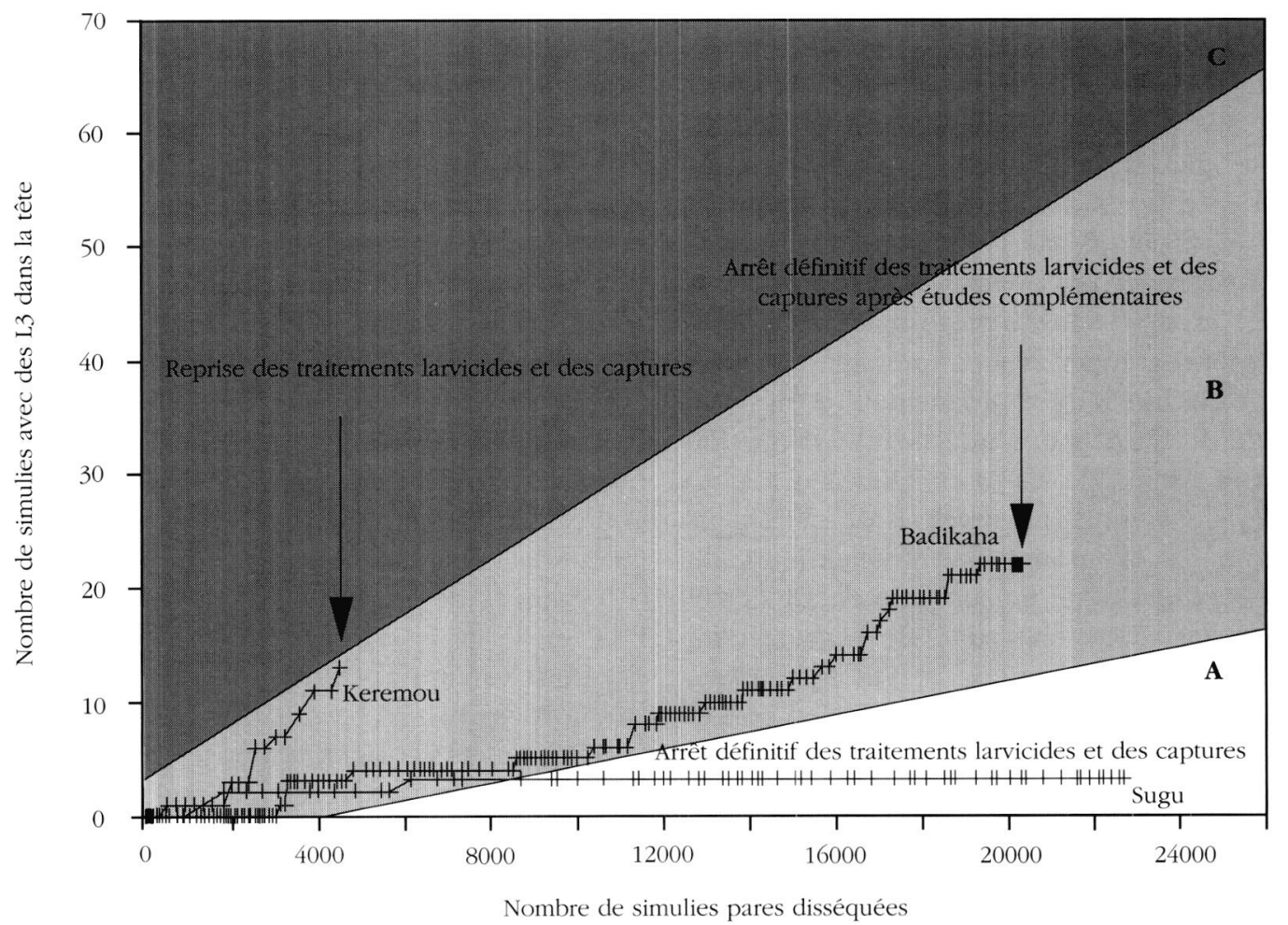

Fig. 2. - Conséquences d’une situation épidémiologique très satisfaisante (Sugu), acceptable (Badikaha) et médiocre (Kérémou) sur les résultats du post-traitement. A : infectivité inférieure à $1 \% 0$; B: infectivité comprise entre 1 et $2 \%$; C : infectivité supérieure à $2 \%$.

\begin{tabular}{|c|c|c|c|c|c|c|}
\hline \multirow{3}{*}{$\begin{array}{l}\text { Points de capture } \\
\text { (rivières) }\end{array}$} & \multirow{3}{*}{$\begin{array}{c}\text { Villages } \\
\text { (distance du point de capture) }\end{array}$} & \multicolumn{5}{|c|}{ Résultats } \\
\hline & & \multicolumn{2}{|c|}{ Début de la lutte } & \multicolumn{3}{|c|}{$\begin{array}{l}\text { Fin de la lutte } \\
\text { (après } 14 \text { à } 16 \text { ans) }\end{array}$} \\
\hline & & $\begin{array}{c}\text { Prév. } \\
\%\end{array}$ & $\begin{array}{l}\text { CMFL } \\
(\mathrm{mf} / \mathrm{b})\end{array}$ & $\begin{array}{c}\text { Prév. } \\
\%\end{array}$ & $\begin{array}{l}\text { CMFL } \\
(\mathrm{mf} / \mathrm{b})\end{array}$ & $\begin{array}{l}\text { Réduction } \\
\text { prévalence }\end{array}$ \\
\hline $\begin{array}{c}\text { Konina } \\
\text { (Banifing IV) }\end{array}$ & $\begin{array}{l}\text { Tyenabougou* } \\
(11 \mathrm{~km})\end{array}$ & 68,4 & 23,1 & 0,0 & 0,00 & $100 \%$ \\
\hline $\begin{array}{c}\text { Badikaha } \\
\text { (Bandama blc) }\end{array}$ & $\begin{array}{l}\text { Fodiolohaha* } \\
(15 \mathrm{~km})\end{array}$ & 59,5 & 22,9 & 0,0 & 0,00 & $100 \%$ \\
\hline $\begin{array}{l}\text { Pont Leraba } \\
\text { (Leraba) }\end{array}$ & $\begin{array}{l}\text { Dangouadougou* } \\
(7 \mathrm{~km})\end{array}$ & 67,9 & 24,3 & 4,8 & 0,12 & $93 \%$ \\
\hline $\begin{array}{c}\text { Nabéré } \\
\text { (Bougouriba) }\end{array}$ & $\begin{array}{l}\text { Nabéré }^{*} \\
(5 \mathrm{~km})\end{array}$ & 82,2 & 36,3 & 1,0 & 0,03 & $99 \%$ \\
\hline $\begin{array}{c}\text { Zambo } \\
\text { (Bougouriba) }\end{array}$ & $\begin{array}{l}\text { Mouvielo* } \\
(12 \mathrm{~km})\end{array}$ & 83,7 & 98,0 & 2,2 & 0,03 & $97 \%$ \\
\hline $\begin{array}{c}\text { Ziou Zabre } \\
\text { (Volta rouge) }\end{array}$ & $\begin{array}{l}\text { Dindergou } \\
(8 \mathrm{~km})\end{array}$ & 78,2 & 74,9 & 1,0 & 0,03 & $98 \%$ \\
\hline $\begin{array}{c}\text { Nangodi } \\
\text { (Volta rouge) }\end{array}$ & $\begin{array}{l}\text { Widennaba } \\
(11 \mathrm{~km})\end{array}$ & 74,5 & 12,9 & 1,8 & 0,10 & $98 \%$ \\
\hline $\begin{array}{c}\text { Zongoïri } \\
\text { (Volta blanche) }\end{array}$ & $\begin{array}{l}\text { Apodabogo } \\
(7 \mathrm{~km})\end{array}$ & 55,8 & 15,7 & 0,0 & 0,00 & $100 \%$ \\
\hline $\begin{array}{c}\text { Loaba } \\
\text { (Volta blanche) }\end{array}$ & $\begin{array}{c}\text { Loaba } \\
(12 \mathrm{~km})\end{array}$ & 76,2 & 53,8 & 0,0 & 0,00 & $100 \%$ \\
\hline $\begin{array}{c}\text { Bukasu } \\
\text { (Oti) }\end{array}$ & $\begin{array}{c}\text { Faré } \\
(8 \mathrm{~km})\end{array}$ & 50,1 & 5,0 & 2,6 & 0,12 & $95 \%$ \\
\hline $\begin{array}{c}\text { Porga } \\
\text { (Oti-Pendjari) }\end{array}$ & $\begin{array}{l}\text { Nandiangou } \\
(19 \mathrm{~km})\end{array}$ & 76,4 & 59,9 & 1,7 & 0,10 & $98 \%$ \\
\hline $\begin{array}{l}\text { Kérémou } \\
\text { (Mékrou) }\end{array}$ & $\begin{array}{l}\text { Gbeniki* } \\
(13 \mathrm{~km})\end{array}$ & 48,8 & 10,4 & 12,1 & 0,70 & $75 \%$ \\
\hline
\end{tabular}

* villages ayant fait l'objet d'une enquête épidémiologique au moins 1 an après le post-traitement.

Tableau I. - Évolution depuis le début de la lutte antivectorielle de la prévalence et de la CMFL dans les villages les plus proches des points de post-traitement. 
post-traitement, les simulies capturées le premier jour de 7 h à 18 h (captures "normalisées ") subissent une dissection complète selon les techniques classiques (Le Berre, 1966). Les simulies capturées les trois jours suivants sont exclusivement réservées aux études de post-traitement. Les captures sont dans ce cas effectuées par des captureurs volontaires, essentiellement aux heures de plus forte agressivité des simulies (captures " en vrac ") et disséquées en vue de déterminer l'âge physiologique de la population et de ne rechercher les larves infectantes de O. volvulus que dans la tête des femelles pares. Il convient de noter que ces deux méthodes d'échantillonnage (captures normalisées et en vrac) donnent des résultats équivalents.

\section{INDICES ENTOMOLOGIQUES ET DÉTERMINATION DU SEUIL D'INFECTIVITÉ DES SIMULIES}

Les indices entomologiques couramment utilisés pour suivre l'impact de la lutte antivectorielle sur la transmission de l'onchocercose sont (Duke, 1968; Walsh et al., 1978) : le Potentiel Annuel de Transmission (PAT), le Taux Annuel de Piqûres (TAP), l'intensité de la transmission, qui s'exprime en nombre de larves infectantes d'onchocerques (L3 tête : nombre de L3 dans la tête) pour 1000 femelles pares et, en ce qui concerne notre étude, l'infectivité, qui est le nombre de simulies infectieuses (avec des L3 tête) pour 1000 pares. Le seuil au-dessous duquel le niveau d'infectivité est jugé acceptable a été déterminé en utilisant le modèle ONCHOSIM (Plaisier et al., 1991b; Remme et al., 1995). Ce seuil est en fait constitué de plusieurs niveaux de décision qui facilitent l'interprétation des données entomologiques (fig. 2). Ces niveaux, sur lesquels est appliquée la méthode séquentielle d'échantillonnage (Armitage et Berry, 1990), sont ceux matérialisés sur la figure 2 et qui se présentent comme suit :

- le niveau correspondant à moins de 1 femelle infectieuse pour 1000 pares indique que les résultats peuvent être considérés comme bons, ce qui permet de confirmer l'arrêt définitif des traitements larvicides et, par voie de conséquence, de mettre fin aux captures. Dans ce cas, le nombre minimum de femelles pares qu'il faut disséquer pour valider les résultats est d'environ 4000 par an pendant les deux années de l'étude;

- à l'opposé, le niveau correspondant à plus de deux femelles infectieuses pour 1000 pares indique que le risque de recrudescence est jugé trop important, imposant ainsi la reprise de la lutte antivectorielle, même avec des effectifs de femelles pares disséquées inférieurs à 4000 ;

- enfin, en cas de situation intermédiaire, la décision d'arrêt des traitements est difficile à prendre sur l'unique base de ce modèle. Les captures et dissections doivent alors se poursuivre en même temps que sont rassemblées des informations complémentaires sur la situation entomo-épidémiologique locale (espèces et densités simulidiennes en présence, origine des simulies infectieuses, phénomènes de migrations de populations humaines, identité des onchocerques décelées, etc.) en vue d'une décision définitive appropriée.

\section{RÉSULTATS}

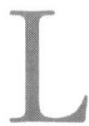

es résultats obtenus (tableau II) sont excellents sur le Bandama blanc à Niaka $(0,57$ femelle infectieuse pour 1000 pares), sur la Léraba au Pont Léraba $(0,51 \%)$, sur la Bougouriba à Nabéré et Zambo $(0,39$ et $0,61 \%)$, sur la Volta rouge à Chache, Ziou-Zabré et Nangodi $(0,49$ et $0,65 \%$ ), sur la Volta blanche à Sugu et Loaba $(0,14$ et $0,33 \% 0)$ et sur l'Oti à Bukasu et Tontionga $(0,40$ et $0,89 \%)$. Sur le Banifing IV, les résultats bruts sur les trois points étudiés ont révélé des valeurs situées bien au delà du seuil admissible d'une femelle infectieuse pour 1000 pares mais les identifications par sondes d'ADN des larves infectantes d'onchocerques, effectuées dans un laboratoire du Programme à Bouaké, en Côte d'Ivoire (Zimmermann et al., 1992), ont montré qu'il s'agissait entièrement d'onchocerques animales appartenant pour la plupart à l'espèce ochengi, parasite des bovins. À Porga, le même phénomène a été partiellement observé ( $62 \%$ d'onchocerques animales), ramenant le taux de femelles infectieuses à 0,49 femelle infectieuse pour 1000 pares.

Les résultats obtenus à Badikaha et à Zongoïri-rapids sont tout juste acceptables (1,08\%0 et 1,16\%). Les résultats obtenus à Kérémou sont par contre au-dessus du seuil d'acceptabilité avec 3,12 femelles infectieuses pour 1000 pares.

\section{DISCUSSION}

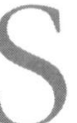
ur les 18 points de post-traitement étudiés, 15 d'entre eux ont, d'emblée, révélé un niveau d'infectivité des simulies compatible avec un arrêt définitif des traitements larvicides sur les bassins concernés. Les enquêtes épidémiologiques réalisées par la suite dans certains des villages de première ligne (tableau I) ont d'ailleurs confirmé le bien-fondé d'une telle décision, démontrant ainsi la justesse des critères entomo-épidémiologiques proposés pour l'arrêt des traitements larvicides. Les études de posttraitement où le seuil d'infectivité était supérieur à 1 


\begin{tabular}{|c|c|c|c|c|c|c|}
\hline $\begin{array}{l}\text { Points de capture } \\
\text { (rivières) }\end{array}$ & Période de capture & $\begin{array}{l}\text { Fem. pares } \\
\text { disséquées }\end{array}$ & $\begin{array}{c}\text { Femelles } \\
\text { infectieuses } \\
(\text { Onchocerca } \mathrm{sp} .)\end{array}$ & $\begin{array}{c}\text { Femelles } \\
\text { infectieuses } \\
\text { (O. volvulus) }\end{array}$ & $\begin{array}{l}\text { Infect. pour } \\
1000 \text { pares }\end{array}$ & Remarques \\
\hline $\begin{array}{c}\text { Kon., Kouo, K.radier } \\
\text { (Banifing IV) }\end{array}$ & de 06/92 à 12/93 & 27246 & 243 & 0 & 0 & $\begin{array}{l}100 \% \text { non } \\
\text { O. volvulus }\end{array}$ \\
\hline $\begin{array}{c}\text { Niaka } \\
\text { (Bandama blanc) }\end{array}$ & de $07 / 93$ à $10 / 94$ & 18369 & 17 & 13 & 0,71 & bons résultats \\
\hline $\begin{array}{c}\text { Badikaha } \\
\text { (Bandama blanc) }\end{array}$ & de $01 / 91$ à $09 / 92$ & 20335 & 22 & non identifiés* & 1,08 & acceptable (migrants) \\
\hline $\begin{array}{c}\text { Pont Léraba } \\
\text { (Léraba) }\end{array}$ & de $01 / 91$ à $02 / 93$ & 21679 & 11 & non identifiés* & 0,51 & bons résultats \\
\hline $\begin{array}{c}\text { Nabéré } \\
\text { (Bougouriba) }\end{array}$ & de $07 / 90$ à $08 / 91$ & 20088 & 8 & non identifiés* & 0,39 & bons résultats \\
\hline $\begin{array}{c}\text { Zambo } \\
\text { (Bougouriba) }\end{array}$ & de $06 / 91$ à $12 / 91$ & 17866 & 11 & non identifiés* & 0,61 & bons résultats \\
\hline $\begin{array}{c}\text { Chache } \\
\text { (Volta noire) }\end{array}$ & de $01 / 94$ à $06 / 94$ & 6880 & 1 & 0 & 0 & bons résultats \\
\hline $\begin{array}{l}\text { Ziou Zabre } \\
\text { (Volta rouge) }\end{array}$ & de $07 / 90$ à $12 / 91$ & 22391 & 11 & non identifiés* & 0,49 & bons résultats \\
\hline $\begin{array}{c}\text { Nangodi } \\
\text { (Volta rouge) }\end{array}$ & de 07/90 à 12/91 & 39739 & 26 & non identifiés* & 0,65 & bons résultats \\
\hline $\begin{array}{c}\text { Sugu } \\
\text { (Volta blanche) }\end{array}$ & de 01/94 à 06/94 & 21078 & 3 & 3 & 0,14 & bons résultats \\
\hline $\begin{array}{c}\text { Zongoïri } \\
\text { (Volta blanche) }\end{array}$ & de $07 / 90$ à $01 / 92$ & 33515 & 39 & non identifiés* & 1,16 & acceptable (migrants) \\
\hline $\begin{array}{c}\text { Loaba } \\
\text { (Volta blanche) }\end{array}$ & de 10/90 à 10/91 & 3026 & 1 & non identifiés* & 0,33 & $\begin{array}{l}\text { bons résultats } \\
\text { peu de simulies }\end{array}$ \\
\hline $\begin{array}{c}\text { Bukasu } \\
\text { (Oti) }\end{array}$ & de 01/92 à 02/94 & 7389 & 6 & 3 & 0,40 & bons résultats \\
\hline $\begin{array}{l}\text { Tontionga } \\
\text { (Oti) }\end{array}$ & de 01/94 à 09/94 & 20278 & 30 & 18 & 0,89 & bons résultats \\
\hline $\begin{array}{c}\text { Porga } \\
\text { (Oti-Pendjari) }\end{array}$ & de $01 / 92$ à $12 / 93$ & 16316 & 21 & 8 & 0,49 & $\begin{array}{l}62 \% \text { non } \\
\text { O. volvulus }\end{array}$ \\
\hline $\begin{array}{l}\text { Kérémou } \\
\text { (Mékrou) }\end{array}$ & de $07 / 92$ à $08 / 92$ & 4165 & 19 & 13 & 3,12 & $\begin{array}{l}\text { reprise des } \\
\text { traitements }\end{array}$ \\
\hline
\end{tabular}

* le laboratoire d'identification des larves infectantes d'onchocerques de l'OCP n'a été opérationnel qu'à partir de 1992.

Tableau II. - Résultats des études entomologiques de post-traitement de1990 à 1994

ont également été riche d'enseignements car elles ont permis de prendre des actions immédiates en vue d'éviter une éventuelle recrudescence de la maladie. Ainsi à Badikaha, l'OCP a réalisé des enquêtes sociologiques et épidémiologiques qui ont montré que certains villages du bassin du Bandama blanc avaient été récemment colonisés par des migrants venant pour la plupart du sud de la Côte d'Ivoire, une zone d'extension du Programme où les épandages larvicides n'ont commencé que depuis 1979. Un traitement à l'ivermectine, un microfilaricide désormais distribué à large échelle dans les zones d'extension du Programme (Remme et al., 1990b), a alors été institué dans tous les villages du bassin afin d'améliorer la situation épidémiologique et d'éviter une reprise des traitements larvicides (fig. 2). À Zongoïri-rapids, une situation similaire due à des pêcheurs migrants infectés venus du sud du Ghana a été mise en évidence. Après le départ des migrants, traités auparavant à l'ivermectine, le taux d'infectivité, qui était de $1,76 \%$, est passé à $0,35 \%$, valeur tout à fait compatible avec un arrêt définitif des traitements larvicides (le chiffre de 1,16\% figurant dans le tableau II correspond à l'indice calculé sur l'ensemble des simulies disséquées). Enfin, à Kérémou où les critères épidé- miologiques n'étaient pas remplis de façon totalement satisfaisante après 15 ans de lutte antivectorielle, les premiers résultats de l'étude de post-traitement ne laissaient d'autre alternative que de reprendre sans tarder les épandages d'insecticides (fig. 2). Cela fut fait dès le mois de septembre de la même année, conjointement avec de nouvelles enquêtes épidémiologiques qui permirent de détecter puis de traiter à l'ivermectine les villages où les prévalences restaient encore élevées.

\section{CONCLUSIONS}

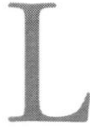
es études entomologiques de post-traitement ont permis d'arrêter définitivement les traitements insecticides sur plus de $90 \%$ des bassins de l'aire initiale du Programme sur le Banifing IV, le Bandama blanc, la Léraba, la Bougouriba, le cours supérieur de la Volta noire, la Volta rouge, la Volta blanche, l'Oti et la Pendjari. En ce qui concerne la Volta blanche et le Bandama blanc, notons que des enquêtes complémentaires ont dû être entreprises à la suite des résultats fournis par le post-traitement et un traitement ponctuel à l'ivermectine a permis peu 
après de procéder à une cessation définitive des épandages insecticides. Cela n'a pas été le cas à Kérémou sur la Mékrou où les résultats ont clairement démontré qu'un arrêt définitif des traitements larvicides était encore prématuré.

De ces résultats et en particulier des actions qui s'en sont suivies, trois conclusions majeures peuvent être tirées :

1) le niveau du seuil d'infectivité de post-traitement proposé par le modèle ONCHOSIM (1 femelle infectieuse pour 1000 pares) est conforté par les résultats de cette étude. En effet, aucune recrudescence de l'infection onchocerquienne n'a jusqu'à présent été signalée sur les bassins où un arrêt définitif des traitements larvicides a été décidé.

2) cette précédente constatation est une preuve supplémentaire que les critères entomo-épidémiologiques retenus pour l'arrêt des traitements larvicides sont parfaitement justifiés. En effet, la seule rivière où les traitements ont repris était située dans une zone où les prévalences n'étaient pas encore totalement satisfaisantes (12,1\% à Gbéniki situé à $13 \mathrm{~km}$ des rives de la Mékrou).

3) enfin, les deux points de Badikaha et Zongoïri montrent clairement les risques que représentent les migrations de populations humaines encore infectées, d'où la nécessité de poursuivre, dans les zones sensibles, une surveillance épidémiologique attentive pour éviter tout risque de recrudescence de la transmission de l'onchocercose.

La validité des critères entomo-épidémiologiques d'arrêt des traitements larvicides fait que ces études de post-traitement ne seront pas renouvelées dans les zones d'extension du Programme. Les résultats auraient été de toutes façons très difficiles à interpréter car ces zones sont traitées également à l'ivermectine, un microfilaricide qui a un impact non négligeable sur la transmission de l'onchocercose (Alley et al., 1994).

\section{REMERCIEMENTS}

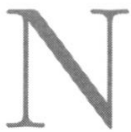
ous remercions tout particulièrement le $\mathrm{Dr}$ E.M. Samba, ancien Directeur de l'OCP et Directeur Régional de l'OMS pour l'Afrique, ainsi que le Dr. J.H.F. Remme de l'OMS/TDR, pour leur soutien constant et leurs précieux conseils apportés lors de la rédaction de ce manuscrit. Nos remerciements s'adressent également aux entomologistes médicaux, techniciens entomologistes, auxiliaires de laboratoire, chauffeurs et captureurs qui, par le sérieux de leur travail, ont été les artisans de ces études de post-traitement, en particulier feu le Dr. Quillévéré, chef de l'unité de lutte antivectorielle du
Programme jusqu'en 1994, le Dr. P. Guillet et Mr. G. Zerbo, chefs de zone respectivement jusqu'en 1992 et 1994, les chefs de secteurs, Messieurs S. Sowah, M. Sarr, les Docteurs A.K. Diallo et M. Kassambara, les chefs de sous-secteurs et techniciens entomologistes Messieurs P. Nikiéma, S. Raogo, G. Nigbeï, T. Osei, M. Agbossou, A. Amadou, F. Guéguédégbo, B. Birrégah et M. Goné. Nous remercions enfin tout particulièrement le Docteur L. Toé, responsable du laboratoire des ADN de l'OCP à Bouaké (Côte d'Ivoire) pour les identifications des larves infectantes d'onchocerques.

\section{RÉFÉRENCES}

Alley E.S., Plaisier A.P., Bohtin B.A., Dadzie K.Y., Remme J., Zerbo G. \& SAmba E.M. The impact of five years of annual ivermectin treatment on skin microfilarial loads in the onchocerciasis focus of Asubende, Ghana. Transactions of the Royal Society of Tropical Medicine and Hygiene, 1994, 88, 581-584.

Anonyme Expert Advisory Committee, Eleventh session, annual internal technical review meeting, Ouagadougou. OCP/EAC 11.4, WHO Mimeographed Document OCP/DIR/90/001, 1990, 32 p.

Armitage P. \& Berry G. Statistical methods in medical research. Ed. Blackwell Scientific Publications, Scd edition, 1990, $559 \mathrm{p}$.

DukE B.O.L. Studies on factors influencing the transmission of onchocerciasis. IV. The biting cycles, infective biting density and transmission potential of "forest Simulium damnosum". Annals of Tropical Medicine and Parasitology, 1968, 62, 95-106.

Hougard J.-M., Poudiougo P., Zerbo D.G., Meyer R., Guillet P., Agoua H., A. Sékétéli A., Akpoboua L.K.B., Sowah S., SAmba E.M. \& Quillévéré D. La lutte contre les vecteurs de l'onchocercose en Afrique de l'Ouest : description d'une logistique appropriée à un programme de santé publique de grande envergure. Cabiers santé, 1994, 4, 389-398.

Le Berre R. Contribution à l'étude biologique et écologique de Simulium damnosum. Théobald, 1903 (Diptera : Simuliidae). Édition de l'ORSTOM, 1966, mém. n 17, 204 p.

Philippon B. Étude de la transmission d'Onchocerca volvulus Leuckart, 1893 par Simulium damnosum Théobald, 1903 (Diptera : Simuliidae) en Afrique Tropicale. Travaux et Documents de l'ORSTOM, 1977, n 63, 308 p.

Philippon B., Remme J.H.F., Walsh J.F., Guillet P. \& Zerbo D.G. Entomological results of vector control in the Onchocerciasis Control Programme. Acta Leidensia, 1990, 59, 79-94.

Plaisier A.P., Van Oortmarssen G., Habbema J.D.F., Remme J. \& AlLEy E.S. ONCHOSIM, a simulation model for the transmission and control of onchocerciasis. Computer Methods and Programs in Biomedicine, 1990, 31, 43-56. 
Plaisier A.P., Van Oortmarssen G., Remme J. \& Habbema J.D.F. The reproductive lifespan of Onchocerca volvulus in West African savanna. Acta Tropica, 1991a, 48, 271284.

Plaisier A.P., Van Oortmarssen G., Remme J., Alley E.S. \& HaввEMA J.D.F. The risk and dynamics of onchocerciasis recrudescence after vector control. Bulletin of the World Health Organization, 1991b, 69, 169-178.

QuilléVÉRÉ D. Contribution à l'étude des caractéristiques taxonomiques, bioécologiques et vectrices des membres du complexe Simulium damnosum présents en Côte d'Ivoire. Paris, ORSTOM, Travaux et Documents de l'ORSTOM, 1979, n 109, 304 p.

Remme J.H.F., Alley E.S. \& Plaisier A.P. Estimation and prediction in tropical disease control : the example of onchocerciasis in epidemic models : their structure and relation to data. In : D. Mollison, Ed. Cambridge University Press, Cambridge, 1995, 377-399.

Remme J., Ba O., Dadzie K.Y. \& Karam M. A force of infection model for onchocerciasis and its application in the epidemiological evaluation of the Onchocerciasis Control Programme in the Volta River basin area. Bulletin of the World Health Organization, 1986, 64 (5), 667-681.

Remme J., De Sole G. \& Van Oortmarssen G. The predicted and observed decline in the prevalence and intensity of onchocerciasis infection during 14 years of successful vector control. Bulletin of the World Health Organization, 1990a, 68, 331-339.

Remme J., De Sole G., Dadzie K.Y., Alley E.S., Baker R.H.A., Hbbema J.D.F., Plaisier A.P., Van Oortmarssen G.J. \& SAMBA E.M. Large scale ivermectin distribution and its epidemiological consequences. Acta Leidensia, 1990b, 59, 177-191.

Walsh J.F., Davies J.B., Le Berre R. \& Garms R. Standardization of criteria for assessing the effect of Simulium control in onchocerciasis control programme. Transactions of the Royal Society of Tropical Medicine and Hygiene, 1978, 72, 675-676.

Zimmermann P.A., ToÉ L. \& Unnash T.R. Rational design of oligonucleotide probes applied to differentiation of Onchocerca volvulus and Oncbocerca ochengi in Northern Côte d'Ivoire. American Journal of Tropical Medicine and Hygiene, 1992, 47, 235-236.

Accepté le 22 avril 1995 